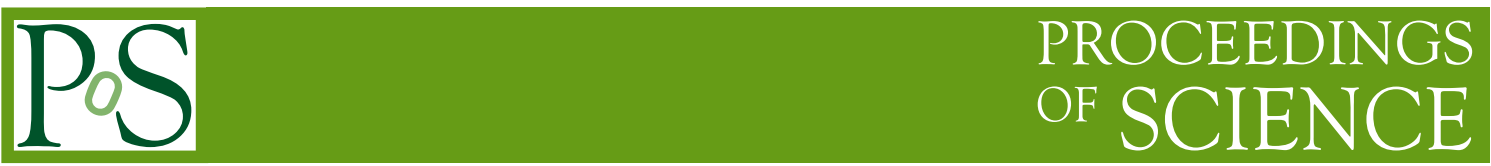

\title{
Double hybrid inflation and gravitational waves
}

\section{G. Lazarides*}

School of Electrical and Computer Engineering, Faculty of Engineering, Aristotle University of Thessaloniki, Thessaloniki 54124, Greece

E-mail: lazaridedeng.auth.gr

\section{Panagiotakopoulos}

School of Rural and Surveying Engineering, Faculty of Engineering, Aristotle University of Thessaloniki, Thessaloniki 54124, Greece

E-mail: costapan@eng.auth.gr

A double hybrid inflationary scenario in non-minimal supergravity which can predict values of the tensor-to-scalar ratio up to about $5 \times 10^{-2}$ is presented. Larger values of this ratio would require unacceptably large running of the scalar spectral index. The underlying supersymmetric particle physics model possesses, for the chosen values of the parameters, practically two inflationary paths, the trivial and the semi-shifted one. The trivial path is stabilized by supergravity and supports a first stage of inflation with a limited number of e-foldings. The tensor-to-scalar ratio can become appreciable with the scalar spectral index remaining acceptable, as a result of the competition between the relatively mild supergravity and the strong radiative corrections to the inflationary potential. The additional number of e-foldings required for solving the puzzles of hot big bang cosmology are generated by a second stage of inflation along the semi-shifted path. This is possible only because the semi-shifted path is almost orthogonal to the trivial one and, thus, not affected by the strong radiative corrections on the trivial path and also because the supergravity effects remain mild. The model predicts the formation of an unstable network of open cosmic strings connecting monopoles to antimonopoles. This network decays to gravity waves well before recombination leading to possibly detectable signatures in future space-based laser interferometer gravitational-wave detectors.

18th International Conference From the Planck Scale to the Electroweak Scale 25-29 May 2015

Ioannina, Greece

\footnotetext{
*Speaker.
} 


\section{Introduction}

The recent results of BICEP2 [1] on the B-mode in the polarization of the cosmic microwave background radiation (CMBR) at degree angular scales indicate that inflationary scenarios may have to face a new challenge. Namely, they should be able to accommodate appreciable values of the tensor-to-scalar ratio $r$, since a B-mode could be due to the production of gravitational waves during inflation. Although $r$ seems [2] to be smaller than initially claimed due to a possible underestimation of the foreground from Galactic polarized-dust emission, values of $r \sim 0.01$ cannot be excluded [3,4]. The most recent joint analysis [4] of the Planck and BICEP2 data yields $r \lesssim 0.12$ at $95 \%$ confidence level.

Supersymmetric (SUSY) hybrid inflation [5] - for a review see e.g. Ref. [6] - is undoubtedly one of the most promising inflationary scenarios. In its simplest realization, though, it suffers from some problems. The grand unified theory (GUT) gauge symmetry is spontaneously broken only at the end of inflation and, thus, if magnetic monopoles are predicted by this breaking, they are copiously produced [7] leading to a cosmological catastrophe. Also, although accurate measurements [8] imply that the scalar spectral index $n_{\mathrm{s}}$ is clearly lower than unity, this scenario gives [9] values very close to unity or even larger than unity within minimal supergravity (SUGRA).

These problems can be solved within a two stage variant of SUSY hybrid inflation with minimal SUGRA, known as standard-smooth hybrid inflation scenario [10]. The cosmological scales exit the horizon during the first stage of inflation, which is of the standard hybrid type, along a trivial classically flat path on which the gauge group is unbroken. Restricting the number of e-foldings during this stage, we can achieve adequately low values of $n_{\mathrm{s}}$. The extra e-foldings needed for solving the horizon and flatness problems of hot big bang cosmology are generated by a second stage of inflation along a classically non-flat valley of minima, where the gauge group is broken. Consequently, magnetic monopoles are produced only at the end of the first inflationary stage, but are adequately diluted by the second stage. Note, in passing, that the idea of a two stage inflation has been used [11] in the past for solving the initial value problem of hybrid inflation.

This scenario was realized within an extended SUSY Pati-Salam (PS) particle physics GUT model with only renormalizable interactions, which was constructed [12] for a very different reason. Namely, the simplest SUSY PS model predicts [13] exact Yukawa unification [14] and, with universal boundary conditions, yields unacceptable values of the $b$-quark mass. In the extended model, Yukawa unification is naturally and moderately violated and this problem is solved.

Here, we will show [15] that a reduced version of this extended SUSY PS model based on the left-right symmetric gauge group $G_{\mathrm{LR}}=S U(3)_{c} \times S U(2)_{\mathrm{L}} \times S U(2)_{\mathrm{R}} \times U(1)_{B-L}$ can also yield a two stage inflationary scenario which can predict values of $r$ up to about 0.05 together with acceptable values of $n_{\mathrm{s}}$. Larger values of $r$ would require unacceptably large running of $n_{\mathrm{s}}$. The first stage occurs along the trivial path, stabilized by SUGRA, and our present horizon undergoes a limited number of e-foldings. The obtained values of $r$ can be appreciable thanks to the presence of strong radiative and relatively mild SUGRA corrections to the inflationary potential. The second stage occurs on the so-called semi-shifted path [16], where $U(1)_{B-L}$ is unbroken, and generates the extra e-foldings required. This is possible since the SUGRA corrections on the semi-shifted path also remain mild and this path, for the parameters chosen, is almost orthogonal to the trivial one and, thus, not affected by the strong radiative corrections on the trivial path. 
After the termination of the first stage of inflation, $S U(2)_{\mathrm{R}}$ breaks spontaneously to a $U(1)$ subgroup. This leads to the production of magnetic monopoles. The spontaneous breaking of a linear combination of this $U(1)$ and $U(1)_{B-L}$ at the end of the second inflationary stage leads to the formation of open cosmic strings connecting these monopoles to antimonopoles. At later times, the monopoles enter the post-inflationary horizon and the string-monopole system decays into gravity waves well before recombination without affecting the CMBR. The resulting gravity waves, however, may be measurable in the future space-based laser interferometer detectors.

\section{The model in global SUSY}

The reduced version of the extended SUSY PS model of Ref. [12] which we will use here is based on the left-right symmetric gauge group $G_{\mathrm{LR}}=S U(3)_{c} \times S U(2)_{\mathrm{L}} \times S U(2)_{\mathrm{R}} \times U(1)_{B-L}$, which is a subgroup of the PS gauge group. The superfields which are relevant for inflation are a conjugate pair of Higgs superfields $H$ and $\bar{H}$ in the $(1,1,2)_{1}$ and $(1,1,2)_{-1}$ representations of $G_{\mathrm{LR}}$, respectively, causing the breaking of $G_{\mathrm{LR}}$ to the standard model gauge group $G_{\mathrm{SM}}$, a gauge singlet $S$, and a pair of superfields $\Phi, \bar{\Phi}$ in the $(1,1,3)_{0}$ representation of $G_{\mathrm{LR}}$. The vacuum expectation value (VEV) $\langle\Phi\rangle$ of $\Phi$ breaks $G_{\mathrm{LR}}$ to $G_{\mathrm{SM}} \times U(1)_{B-L}$.

The superpotential relevant for inflation is

$$
W=\kappa S\left(M^{2}-\Phi^{2}\right)-\gamma S H \bar{H}+m \Phi \bar{\Phi}-\lambda \bar{\Phi} H \bar{H} .
$$

The parameters $M, m$ are superheavy masses, while $\kappa, \gamma, \lambda$ are dimensionless constants. All these parameters but one can be made real and positive by rephasing the superfields. For definiteness, we choose the remaining complex parameter to be real and positive too.

The resulting F-term scalar potential is

$$
V_{F}^{0}=\left|\kappa\left(M^{2}-\Phi^{2}\right)-\gamma H \bar{H}\right|^{2}+|m \bar{\Phi}-2 \kappa S \Phi|^{2}+|m \Phi-\lambda H \bar{H}|^{2}+|\gamma S+\lambda \bar{\Phi}|^{2}\left(|H|^{2}+|\bar{H}|^{2}\right) .
$$

From $V_{F}^{0}$ and the vanishing of the D-terms, which implies that $\bar{H}^{*}=e^{i \theta} H$, one finds [16] two distinct continua of SUSY vacua:

$$
\begin{gathered}
\Phi=\Phi_{+}, \quad \bar{H}^{*}=H, \quad|H|=\sqrt{\frac{m \Phi_{+}}{\lambda}} \quad(\theta=0), \quad \bar{\Phi}=S=0, \\
\Phi=\Phi_{-}, \quad \bar{H}^{*}=-H, \quad|H|=\sqrt{\frac{-m \Phi_{-}}{\lambda}} \quad(\theta=\pi), \quad \bar{\Phi}=S=0,
\end{gathered}
$$

where

$$
\Phi_{ \pm} \equiv \pm M\left(\sqrt{1+\left(\frac{\gamma m}{2 \kappa \lambda M}\right)^{2}} \mp \frac{\gamma m}{2 \kappa \lambda M}\right) .
$$

The model generally possesses [16] three flat directions: $(i)$ The usual trivial path at

$$
\Phi=\bar{\Phi}=H=\bar{H}=0 \quad \text { with } \quad V_{F}^{0}=V_{\mathrm{tr}} \equiv \kappa^{2} M^{4},
$$

where $G_{\mathrm{LR}}$ is unbroken. (ii) The new shifted path at

$$
\Phi=-\frac{\gamma m}{2 \kappa \lambda}, \quad \bar{\Phi}=-\frac{\gamma}{\lambda} S, \quad H \bar{H}=\frac{\kappa \gamma\left(M^{2}-\Phi^{2}\right)+\lambda m \Phi}{\gamma^{2}+\lambda^{2}}
$$


with

$$
V_{F}^{0}=V_{\mathrm{nsh}} \equiv \kappa^{2} M^{4}\left(\frac{\lambda^{2}}{\gamma^{2}+\lambda^{2}}\right)\left(1+\frac{\gamma^{2} m^{2}}{4 \kappa^{2} \lambda^{2} M^{2}}\right)^{2} .
$$

This path supports new shifted hybrid inflation [17] with $G_{\mathrm{LR}}$ broken to $G_{\mathrm{SM}}$. (iii) The semi-shifted path, which exists only for $M^{2}>m^{2} / 2 \kappa^{2}$, at

$$
\begin{gathered}
\Phi= \pm M \sqrt{1-\frac{m^{2}}{2 \kappa^{2} M^{2}}}, \quad \bar{\Phi}=\frac{2 \kappa \Phi}{m} S, \quad H=\bar{H}=0 \\
\text { with } \quad V_{F}^{0}=V_{\text {ssh }} \equiv m^{2} M^{2}\left(1-\frac{m^{2}}{4 \kappa^{2} M^{2}}\right) .
\end{gathered}
$$

It yields semi-shifted hybrid inflation [16] with $U(1)_{B-L}$ unbroken.

We take $M^{2}>m^{2} / 2 \kappa^{2}$ and, thus, the semi-shifted path exists and, as one can show [16], always lies lower than the trivial and the new shifted one. We also take $\kappa \sim 1, \gamma \ll \lambda \ll \kappa$, and $m \ll M$, so that the new shifted path (for $|S|<1$ ) essentially coincides with the trivial one and, thus, plays no independent role in our scheme.

\section{The first stage of inflation}

The first stage of inflation takes place along the trivial path, which, for large values of the canonically normalized inflaton, is stabilized by the SUGRA corrections. Although the number of e-foldings is limited, all the cosmological scales exit the horizon during this inflationary stage. Strong radiative and relatively mild SUGRA corrections to the inflationary potential then guarantee appreciable values of $r$ together with acceptable values of $n_{\mathrm{s}}$.

We adopt the Kähler potential

$$
K=-\ln \left(1-|S|^{2}\right)-\ln \left(1-|\bar{\Phi}|^{2}\right)+|\Phi|^{2}+|H|^{2}+|\bar{H}|^{2}-2 \ln \left(-\ln \left|Z_{1}\right|^{2}\right)+\left|Z_{2}\right|^{2} .
$$

The two extra $G_{\mathrm{LR}}$ singlets $Z_{1}$ and $Z_{2}$ included in $K$ do not enter the superpotential. The resulting F-term potential in SUGRA is found to be

$$
V_{F}=\left[\sum_{i}\left|W_{X_{i}}+K_{X_{i}} W\right|^{2} K_{X_{i} X_{i}^{*}}^{-1}-3|W|^{2}\right] e^{K},
$$

where the sum is over all the fields $S, \Phi, \bar{\Phi}, H, \bar{H}, Z_{1}, Z_{2}$ and a subscript $X_{i}$ denotes derivation with respect to $X_{i}$. The values of $Z_{1}$ and $Z_{2}$ are fixed [18] by anomalous D-terms. Note that the superfields $S, \bar{\Phi}, Z_{1}$ have no-scale type Kähler potentials which, in view of the relation

$$
\left|K_{Z_{1}}\right|^{2} K_{Z_{1} Z_{1}{ }^{*}}^{-1}=2,
$$

guarantee the exact flatness of the potential along the trivial path [18] and its approximate flatness on the semi-shifted one for $Z_{2}=0$. The relation

$$
\left|K_{Z_{2}}\right|^{2} K_{Z_{2} Z_{2}{ }^{*}}^{-1}=\left|Z_{2}\right|^{2} \equiv \beta
$$

then implies - cf. Ref. [18] - that, for $Z_{2} \neq 0$, the complex inflatons $S$ and $\bar{\Phi}$ (approximately) for the two paths, respectively, acquire masses squared proportional to $\beta$. 
Using the symmetries, we can rotate $S$ and $H$ on the real axis. The fields $\Phi, \bar{\Phi}, \bar{H}$ remain in general complex. For simplicity, we restrict them on the real axis too. This will not influence our results in any essential way since these fields are anyway real in the vacuum and on all the flat directions. Also, we can show that, everywhere on the trivial and the semi-shifted inflationary paths, the mass-squared matrices of the imaginary parts of the fields do not mix with the masssquared matrices of their real parts and, during both inflations, have positive eigenvalues in the directions perpendicular to these paths. So there is no instability in the direction of the imaginary parts of the fields which are orthogonal to these inflationary paths. Moreover, as we can prove, both the trivial and the semi-shifted inflationary paths are destabilized with the fields developing real values.

The canonically normalized real scalar fields $\sigma, \phi, \bar{\phi}, h, \bar{h}$ corresponding to the Kähler potential in Eq. (3.1) are given by - cf. Ref. [18] -

$$
S=\tanh \frac{\sigma}{\sqrt{2}}, \quad \Phi=\frac{\phi}{\sqrt{2}}, \quad \bar{\Phi}=\tanh \frac{\bar{\phi}}{\sqrt{2}}, \quad H=\frac{h}{\sqrt{2}}, \quad \bar{H}=\frac{\bar{h}}{\sqrt{2}} .
$$

We evaluate the potential $V_{F}$ with the factor $\exp \left[-2 \ln \left(-\ln \left|Z_{1}\right|^{2}\right)+\left|Z_{2}\right|^{2}\right]$ absorbed into redefined parameters $\kappa, \gamma, m$, and $\lambda$ and find

$$
\begin{aligned}
V_{F}= & {\left[A_{1}^{2} \cosh ^{2} \frac{\bar{\phi}}{\sqrt{2}}-A_{2}^{2} \sinh ^{2} \frac{\bar{\phi}}{\sqrt{2}}+\beta A_{3}^{2}+A_{4}^{2}+A_{5}^{2}+\frac{1}{2}\left(h^{2}+\bar{h}^{2}\right) A_{6}^{2}\right.} \\
& \left.+\frac{1}{2}\left(\phi^{2}+h^{2}+\bar{h}^{2}\right) A_{3}^{2}+\left(\sqrt{2} \phi A_{5}-2 h \bar{h} A_{6}\right) A_{3}\right] e^{\frac{1}{2}\left(\phi^{2}+h^{2}+\bar{h}^{2}\right)} .
\end{aligned}
$$

Here

$$
\begin{aligned}
A_{1} & =\kappa\left(M^{2}-\frac{\phi^{2}}{2}\right)-\frac{\gamma}{2} h \bar{h}, \quad A_{2}=m \frac{\phi}{\sqrt{2}}-\frac{\lambda}{2} h \bar{h}, \\
A_{3} & =A_{1} \sinh \frac{\sigma}{\sqrt{2}} \cosh \frac{\bar{\phi}}{\sqrt{2}}+A_{2} \cosh \frac{\sigma}{\sqrt{2}} \sinh \frac{\bar{\phi}}{\sqrt{2}}, \\
A_{4} & =A_{1} \sinh \frac{\sigma}{\sqrt{2}} \sinh \frac{\bar{\phi}}{\sqrt{2}}+A_{2} \cosh \frac{\sigma}{\sqrt{2}} \cosh \frac{\bar{\phi}}{\sqrt{2}}, \\
A_{5} & =m \cosh \frac{\sigma}{\sqrt{2}} \sinh \frac{\bar{\phi}}{\sqrt{2}}-\sqrt{2} \kappa \phi \sinh \frac{\sigma}{\sqrt{2}} \cosh \frac{\bar{\phi}}{\sqrt{2}},
\end{aligned}
$$

and

$$
A_{6}=\gamma \sinh \frac{\sigma}{\sqrt{2}} \cosh \frac{\bar{\phi}}{\sqrt{2}}+\lambda \cosh \frac{\sigma}{\sqrt{2}} \sinh \frac{\bar{\phi}}{\sqrt{2}} .
$$

On the trivial path $(\phi, \bar{\phi}, h, \bar{h}=0), V_{F}$ becomes

$$
V_{F}=\kappa^{2} M^{4}\left[1+\beta \sinh ^{2} \frac{\sigma}{\sqrt{2}}\right] \text {. }
$$

The mass-squared eigenvalues in the directions which are perpendicular to the trivial path, for $\sinh ^{2}(\sigma / \sqrt{2}) \gg M^{2} / 2$, are

$$
m_{\phi}^{2} \simeq 4 \kappa^{2} \sinh ^{2} \frac{\sigma}{\sqrt{2}}, \quad m_{\bar{\phi}}^{2} \simeq \kappa^{2} M^{4}\left[1+(1+\beta) \sinh ^{2} \frac{\sigma}{\sqrt{2}}\right]
$$


and

$$
m_{\chi_{1}, \chi_{2}}^{2}=\left(\kappa M^{2} \mp \gamma\right)\left[\kappa M^{2}+\left((1+\beta) \kappa M^{2} \mp \gamma\right) \sinh ^{2} \frac{\sigma}{\sqrt{2}}\right],
$$

where $\chi_{1,2}=(h \pm \bar{h}) / \sqrt{2}$. Note that, in particular, the mass-squared formulas in Eq. (3.14) hold for any value of $\sigma$. Thus, for $\gamma<\kappa M^{2}$, the trivial path is stable for large values of $|\sigma|$. However, as $|\sigma|$ decreases, the eigenvalues and eigenstates of the $\phi-\bar{\phi}$ system change. When $\sinh ^{2}(\sigma / \sqrt{2}) \simeq$ $M^{2} / 2+m^{2} / 2 \kappa^{2} M^{2}$, one of these eigenvalues vanishes with $\bar{\phi}$ dominating the corresponding eigenstate. As $\sinh ^{2}(\sigma / \sqrt{2}) \rightarrow M^{2} / 2$, the eigenvalues become opposite to each other with $\phi, \bar{\phi}$ contributing equally to both the eigenstates. A further decrease of $\sinh ^{2}(\sigma / \sqrt{2})$ leads to the domination of the unstable eigenstate by $\phi$. Since $\phi$ must become nonzero to cancel the energy density $\kappa^{2} M^{4}$ on the trivial path, we say that this path is destabilized at $\sigma_{\mathrm{c}}$ with

$$
\sinh ^{2} \frac{\sigma_{\mathrm{c}}}{\sqrt{2}}=\frac{M^{2}}{2} .
$$

To $V_{F}$ on the trivial path we add the dominant one-loop radiative corrections from the $N_{\phi^{-}}$ dimensional supermultiplet $\Phi\left(N_{\phi}=3\right)$ :

$$
V_{r}^{\phi}=\kappa^{2} M^{4}\left(\frac{N_{\phi} \kappa^{2}}{8 \pi^{2}}\right) \ln \frac{2 \tanh ^{2} \frac{\sigma}{\sqrt{2}}}{M^{2}} .
$$

Note that the renormalization scale in these radiative corrections is chosen such that $V_{r}^{\phi}$ vanishes at $|\sigma|=\left|\sigma_{\mathrm{c}}\right|$.

The full inflationary potential $V$ and its derivatives with respect to $\sigma$ (denoted by primes) are:

$$
\begin{gathered}
\frac{V}{\kappa^{2} M^{4}}=1+\beta \sinh ^{2} \frac{\sigma}{\sqrt{2}}+\frac{\delta_{\phi}}{4} \ln \frac{2 \tanh ^{2} \frac{\sigma}{\sqrt{2}}}{M^{2}} \equiv C(\sigma), \\
\frac{V^{\prime}}{\kappa^{2} M^{4}}=\frac{1}{\sqrt{2}} \sinh (\sqrt{2} \sigma)\left(\beta+\frac{\delta_{\phi}}{\sinh ^{2}(\sqrt{2} \sigma)}\right), \\
\frac{V^{\prime \prime}}{\kappa^{2} M^{4}}=\cosh (\sqrt{2} \sigma)\left(\beta-\frac{\delta_{\phi}}{\sinh ^{2}(\sqrt{2} \sigma)}\right), \\
\frac{V^{\prime \prime \prime}}{\kappa^{2} M^{4}}=\sqrt{2} \sinh (\sqrt{2} \sigma)\left(\beta-\frac{\delta_{\phi}}{\sinh ^{2}(\sqrt{2} \sigma)}\right)+\frac{2 \sqrt{2} \delta_{\phi}}{\tanh ^{2}(\sqrt{2} \sigma) \sinh (\sqrt{2} \sigma)}
\end{gathered}
$$

with

$$
\delta_{\phi}=\frac{N_{\phi} \kappa^{2}}{2 \pi^{2}} .
$$

The usual slow-roll parameters for inflation are then

$$
\begin{gathered}
\varepsilon=\frac{1}{2}\left(\frac{V^{\prime}}{\kappa^{2} M^{4}}\right)^{2} \frac{1}{C^{2}(\sigma)}, \quad \eta=\left(\frac{V^{\prime \prime}}{\kappa^{2} M^{4}}\right) \frac{1}{C(\sigma)}, \\
\xi=\left(\frac{V^{\prime}}{\kappa^{2} M^{4}}\right)\left(\frac{V^{\prime \prime \prime}}{\kappa^{2} M^{4}}\right) \frac{1}{C^{2}(\sigma)}=2|\tanh (\sqrt{2} \sigma)| \eta \sqrt{\varepsilon}+\frac{4 \delta_{\phi} \sqrt{\varepsilon}}{C(\sigma) \tanh ^{2}(\sqrt{2} \sigma)|\sinh (\sqrt{2} \sigma)|} .
\end{gathered}
$$


From these expressions, we evaluate $n_{\mathrm{s}}$, its running $\alpha_{\mathrm{s}}$, the tensor-to-scalar ratio $r$, and $V$ :

$$
n_{\mathrm{s}}=1+2 \eta-6 \varepsilon, \quad \alpha_{\mathrm{s}}=16 \eta \varepsilon-24 \varepsilon^{2}-2 \xi, \quad r=16 \varepsilon, \quad V=\frac{3 \pi^{2}}{2} A_{\mathrm{s}} r
$$

where $A_{\mathrm{s}}$ is the scalar power spectrum amplitude.

As a numerical example, take $\sigma_{*}=1.45$ at horizon exit of the pivot scale $k_{*}=0.05 \mathrm{Mpc}^{-1}$, $\kappa=1.7, \beta=0.022$, and $A_{\mathrm{s}}=2.215 \times 10^{-9}$ at the same $k_{*}$ [8]. We then find $M=3.493 \times 10^{-3}$, $C\left(\sigma_{*}\right)=2.2941, \varepsilon=0.00188, \eta=-0.01389, n_{\mathrm{s}}=0.9609, r=0.0301$, and $\alpha_{\mathrm{s}}=-0.01674$. So we can not only be consistent with the latest Planck data [8], but also accommodate large values of $r \sim$ few $\times 10^{-2}$. Note that large values of $r$ require relatively large values of $\varepsilon$, which reduce $n_{\mathrm{S}}$ below unity, but not enough to make it compatible with the data. So large negative values of $\eta$ are needed, which requires that the parenthesis in the formula for $V^{\prime \prime}$ in Eq. (3.19) is dominated by the second term. A similar parenthesis appears in the formula for $V^{\prime}$ in Eq. (3.18) too, but with the two terms added. So both these terms have to be appreciable with the second one being larger, which is possible only for large values of $\kappa$, which controls the radiative corrections on the trivial path. Inflation ends before the system reaches $\sigma_{\mathrm{c}}$ by violating the slow-roll conditions and the obtained number of e-foldings is limited due to the large values of $\varepsilon$ and the fact that $\sigma_{*} \sim 1$.

\section{The second stage of inflation}

We choose, for the rest of the parameters, $m=1.827 \times 10^{-5}, \lambda=0.1$, and $\gamma=10^{-6}$. Numerically, including also the D-terms from $H$ and $\bar{H}$, we find that there exist initial conditions for which, after the first stage of inflation, the energy density approaches $m^{2} M^{2}$ and $\phi^{2} \simeq 2 M^{2}$, $h, \bar{h} \simeq 0, A_{5} \simeq 0$ with $|\sigma| \ll 1$. So the system reaches the semi-shifted path and a second stage of inflation can take place. It is worth noticing that the initial values of the fields which lead to a double inflation scenario, although quite frequent, do not seem to form well-defined connected regions. In other words, the coupled system of differential equations exhibits a 'chaotic' behavior, which means that a slight change of the initial conditions can possibly lead from a double to a single inflation scenario. A similar situation has been encountered [19] even in the simplest SUSY hybrid inflation scenario, where a slight change of initial conditions may ruin inflation.

The potential $V_{F}$ on the semi-shifted path, for $M^{2} \ll \beta$, is found to be

$$
V_{F} \simeq m^{2} M^{2}\left[1+\beta \sinh ^{2} \frac{\bar{\phi}}{\sqrt{2}}\right] \text {. }
$$

Notice the striking similarity of this expression with the expression for $V_{F}$ on the trivial path in Eq. (3.12). So the SUGRA corrections remain relatively mild on the semi-shifted path too. From $A_{5} \simeq 0$, we find that the combination of $S$ and $\bar{\Phi}$ which is the complex inflaton during the second stage of inflation is

$$
\frac{m S+2 \kappa<\Phi>\bar{\Phi}}{\sqrt{m^{2}+4 \kappa^{2} M^{2}}} \simeq \bar{\Phi}
$$

since $\bar{\Phi}$ contributes in this combination $2 \kappa M / m \simeq 650$ times more than $S$.

The mass eigenstates for the $h-\bar{h}$ system during the second stage of inflation are $\chi_{1,2}=$ $(h \pm \bar{h}) / \sqrt{2}$ with masses squared

$$
m_{\chi_{1}, \chi_{2}}^{2}=(\lambda \mp m M)\left[(\lambda \mp(1+\beta) m M) \sinh ^{2} \frac{\bar{\phi}}{\sqrt{2}} \mp m M\right] .
$$


The eigenstate $\chi_{1}$ develops an instability terminating the semi-shifted valley of minima with the critical value $\bar{\phi}_{\mathrm{c}}$ of the real canonically normalized inflaton $\bar{\phi}$ being given by

$$
\sinh ^{2} \frac{\bar{\phi}_{\mathrm{c}}}{\sqrt{2}}=\frac{m M}{\lambda} .
$$

During the second stage of inflation, we include the dominant radiative corrections to the potential, which originate from the $N_{h}$-dimensional superfields $H, \bar{H}\left(N_{h}=2\right)$ and read as

$$
V_{r}^{h} \simeq m^{2} M^{2}\left(\frac{N_{h} \lambda^{2}}{16 \pi^{2}}\right) \ln \frac{\lambda \tanh ^{2} \frac{\bar{\phi}}{\sqrt{2}}}{m M} .
$$

The renormalization scale is chosen so that $V_{r}^{h}=0$ at $|\bar{\phi}|=\left|\bar{\phi}_{\mathrm{c}}\right|$. The radiative corrections from $\Phi$ are neglected since they are relatively very small. This is because $\Phi$ couples to the complex inflaton only through $S$ and the contribution of $S$ to this inflaton is severely suppressed. This is a very important property of the model resulting from the fact that, for the parameters chosen, the semi-shifted path is almost orthogonal to the trivial one. So the very strong radiative corrections on the trivial path, needed for accommodating appreciable values of $r$, do not affect the second stage of inflation. This is crucial since otherwise the semi-shifted path would be too steep to generate the extra e-foldings required.

The number of e-foldings during the second stage of inflation between an initial $\bar{\phi}_{\text {in }}$ and a final $\bar{\phi}_{\mathrm{f}}$ value of the inflaton $\bar{\phi}$ is $N\left(\bar{\phi}_{\mathrm{f}}\right)-N\left(\bar{\phi}_{\mathrm{in}}\right)$, where

$$
N(\bar{\phi}) \simeq \frac{1}{2 \beta \sqrt{1-\left(\delta_{h} / \beta\right)}} \ln \frac{\cosh (\sqrt{2} \bar{\phi})+\sqrt{1-\left(\delta_{h} / \beta\right)}}{\cosh (\sqrt{2} \bar{\phi})-\sqrt{1-\left(\delta_{h} / \beta\right)}}
$$

with $\delta_{h}=N_{h} \lambda^{2} / 4 \pi^{2}$. The termination of inflation is due to the radiative corrections and occurs at $\bar{\phi}=\bar{\phi}_{\mathrm{f}}\left(\left|\bar{\phi}_{\mathrm{f}}\right| \gg\left|\bar{\phi}_{\mathrm{c}}\right|\right)$ with

$$
\cosh \left(\sqrt{2} \bar{\phi}_{\mathrm{f}}\right) \simeq \frac{\delta_{h}}{2}+\sqrt{1+\frac{\delta_{h}^{2}}{4}}
$$

Numerically, we find that, with the chosen values of the model parameters, the first stage gives rise to about 13 e-foldings. So another 38-39 e-foldings (for reheat temperature $T_{\mathrm{r}}=10^{9} \mathrm{GeV}$ ) must be provided by the second stage of inflation, which requires that $\left|\bar{\phi}_{\text {in }}\right| \simeq 0.23$ at the onset of this stage. This can indeed be satisfied in our numerical example as we have shown by extensive numerical calculations. It is actually remarkable that $|\bar{\phi}|$, which at the end of the first inflationary stage is extremely small, manages to attain values of the order of few $\times 10^{-1}$ at the onset of the second stage.

To see this remarkable jump of $\bar{\phi}$ after the end of the first inflationary stage, we depict, in Fig. 1, the evolution of the fields $\sigma$ and $\bar{\phi}$ as functions of the number of e-foldings $N$ starting from the point where the pivot scale $k_{*}=0.05 \mathrm{Mpc}^{-1}$ exits the horizon for a particular choice of initial conditions. Namely, we start with $\sigma=1.45, \bar{\phi}=10^{-3}, \phi=10^{-8}, h=10^{-4}$, and $\bar{h}=1.01 \times 10^{-4}$. All the fields start with zero velocity except for $\sigma$ the initial velocity of which is taken to be $-1.1074 \times 10^{-6}$, which is its actual velocity on the trivial path determined numerically. We observe that $\sigma$ remains above its critical value for about 13 e-foldings. Just before the end of the first inflationary stage, $\sigma$ oscillates four times around zero with appreciable amplitude. When this amplitude falls below 


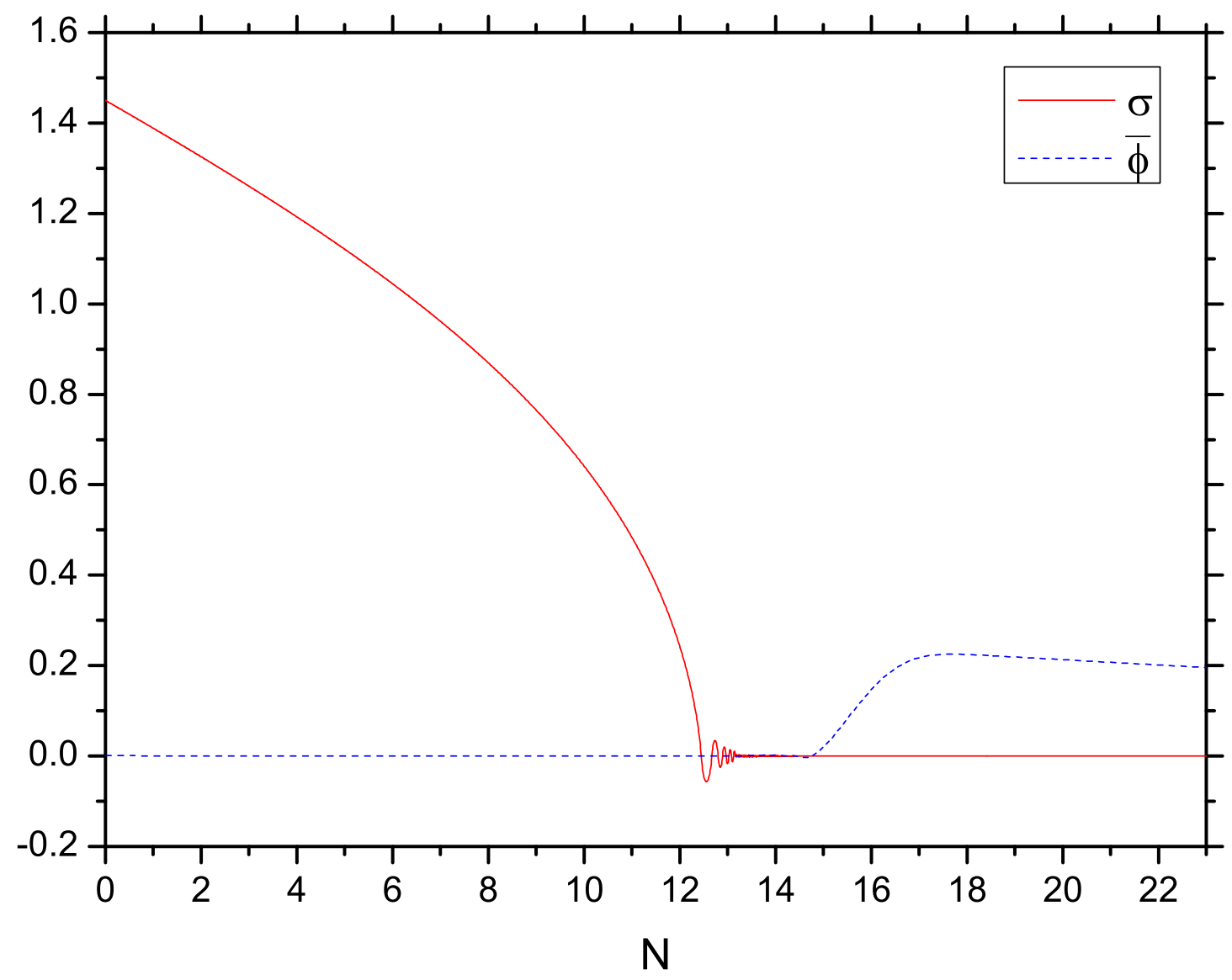

Figure 1: The evolution of $\sigma$ and $\bar{\phi}$ for the case with $r=0.0301$ versus the number $N$ of e-foldings after the horizon exit of the pivot scale $k_{*}$, where we take $\sigma=1.45, \bar{\phi}=10^{-3}, \phi=10^{-8}, h=10^{-4}, \bar{h}=1.01 \times 10^{-4}$, and $d \sigma / d t=-1.1074 \times 10^{-6}$.

the critical value of $\sigma, \phi$ moves to its value on the semi-shifted path and $\bar{\phi}$ starts oscillating slowly with variable amplitudes of order $M$. The size of $\bar{\phi}$ remains small for about $1.7 \mathrm{e}$-foldings before starting its remarkable growth. This field acquires its largest value $\simeq 0.225$ at $N \simeq 17.7$, when the second inflationary stage has already started. For $N \gtrsim 20$, the evolution of $\bar{\phi}$ follows Eq. (4.6) closely.

Allowing for a stronger running of $n_{\mathrm{s}}$, we can achieve larger values of $r$. For example, taking $\sigma_{*}=1.35, \kappa=1.75$, and $\beta=0.037$, we find that $M=3.891 \times 10^{-3}, C\left(\sigma_{*}\right)=2.3479, \varepsilon=0.00314$, $\eta=-0.00844, n_{\mathrm{s}}=0.9643, \alpha_{\mathrm{s}}=-0.03007$, and $r=0.0502$. In addition, we choose $m=3.891 \times$ $10^{-5}, \lambda=0.1$, and $\gamma=10^{-6}$. In this case, the pivot scale suffers about 10 e-foldings during the first inflationary stage and, consequently, approximately another 41-42 e-foldings must be provided by the second stage. This implies that $\left|\bar{\phi}_{\text {in }}\right|$ must lie in the range 0.38-0.40, which is indeed feasible as we verified numerically. In Fig. 2, we depict the evolution of $\sigma$ and $\bar{\phi}$ as functions of the number of e-foldings $N$ again starting from the point where the pivot scale $k_{*}$ exits the horizon, where we make a particular choice of initial conditions shown in the caption of the figure.

Changing the values of the input parameters of the model, we can easily achieve successful 


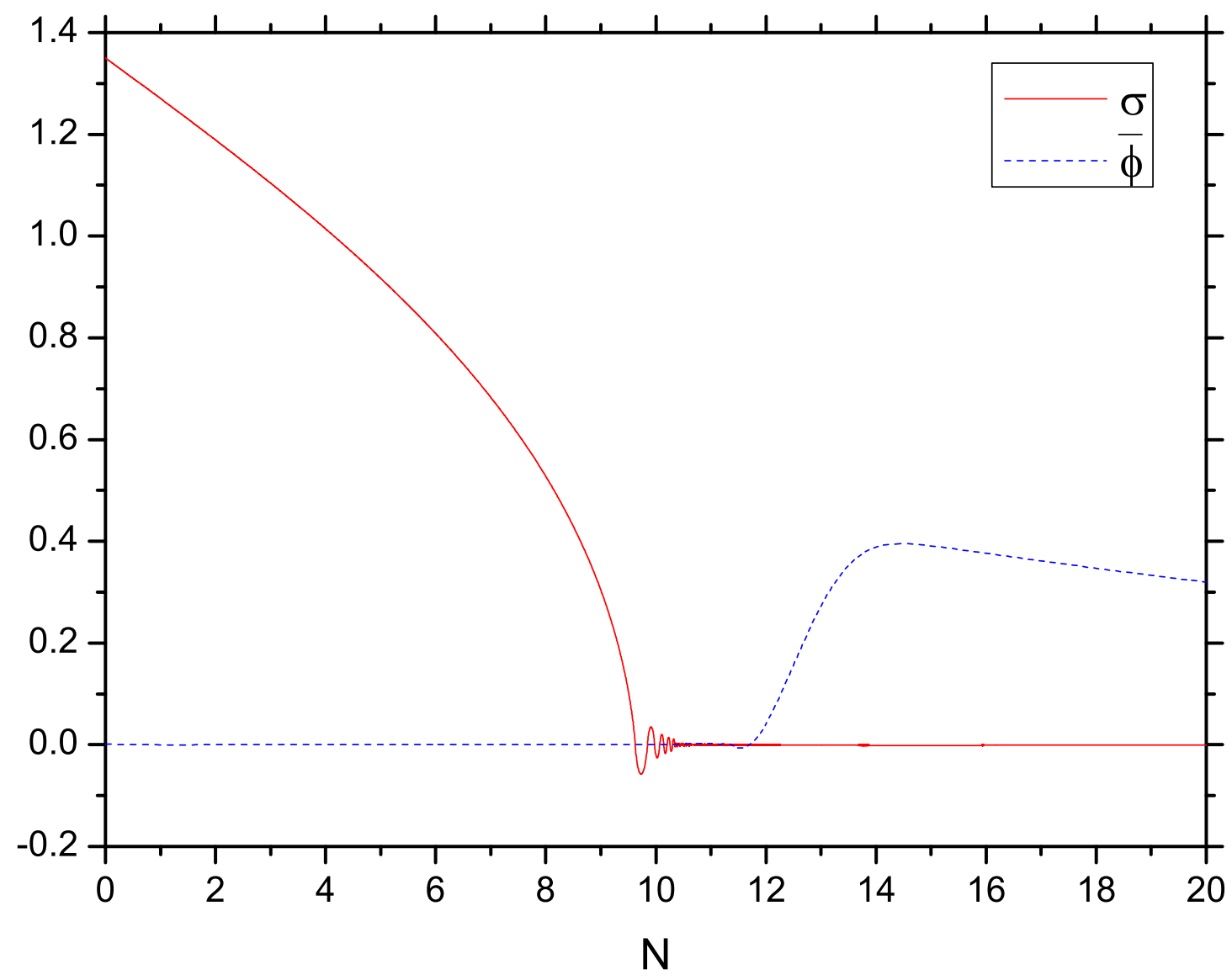

Figure 2: The evolution of $\sigma$ and $\bar{\phi}$ for the case with $r=0.0502$ versus the number $N$ of e-foldings after the horizon exit of the pivot scale $k_{*}$, where we take $\sigma=1.35, \bar{\phi}=10^{-3}, \phi=10^{-8}, h=9 \times 10^{-4}, \bar{h}=$ $9.01 \times 10^{-4}$, and $d \sigma / d t=-1.8523 \times 10^{-6}$.

solutions with smaller values of $r$. Note that there is no particular fine-tuning of the parameters required in our model.

\section{Magnetic monopoles and cosmic strings}

Soon after the end of the first inflationary stage, the system settles on the semi-shifted path and $S U(2)_{\mathrm{R}}$ breaks spontaneously to a $U(1)$ subgroup by the nonzero value of $\Phi$. So magnetic monopoles are formed. An order of magnitude estimate of the mean monopole-antimonopole distance can be obtained as follows. At production, this distance is $p(2 \kappa M)^{-1}$ as determined by the Higgs correlation length with $p \sim 1$ being a geometric factor. In the matter dominated era between the two inflationary stages, this distance is enhanced by a factor $\sim\left(\kappa^{2} M^{4} / m^{2} M^{2}\right)^{1 / 3}$, where $\kappa^{2} M^{4}$ and $m^{2} M^{2}$ are the classical potential energy densities on the trivial and the semi-shifted paths, respectively. The second inflationary stage stretches this distance by a factor $\exp N_{2}$ with $N_{2}$ being the corresponding number of e-foldings which is large but not huge - cf. Ref. [20]. During the damped inflaton oscillations, this distance increases by another factor $\sim\left(m^{2} M^{2} / c\left(T_{\mathrm{r}}\right) T_{\mathrm{r}}^{4}\right)^{1 / 3}$, 
where $T_{\mathrm{r}} \sim 10^{9} \mathrm{GeV}$ is the reheat temperature and $c(T)=\pi^{2} g(T) / 30$ with $g(T)$ being the effective number of massless degrees of freedom at cosmic temperature $T$. In the subsequent radiation dominated period, the monopole-antimonopole distance is multiplied by another factor $\sim T_{\mathrm{r}} / T \sim(4 c(T) / 3)^{1 / 4} T_{\mathrm{r}} \sqrt{t}$, where $t$ is the cosmic time. So this distance, in the radiation dominated period, becomes

$$
\sim\left(\frac{4}{3}\right)^{\frac{1}{4}} c\left(T_{\mathrm{r}}\right)^{-\frac{1}{3}} c(T)^{\frac{1}{4}} p(2 \kappa M)^{-1} e^{N_{2}}\left(\frac{\kappa^{2} M^{4}}{T_{\mathrm{r}}^{4}}\right)^{\frac{1}{3}} T_{\mathrm{r}} \frac{1}{2}^{\frac{1}{2}} .
$$

Equating this distance with the post-inflationary particle horizon $\sim 2 t$, we find the time $t_{\mathrm{H}}$ at which the monopoles enter this horizon:

$$
t_{\mathrm{H}} \sim \frac{p^{2}}{8 \sqrt{3}} c\left(T_{\mathrm{r}}\right)^{-\frac{2}{3}} c\left(T_{\mathrm{H}}\right)^{\frac{1}{2}} e^{2 N_{2}}\left(\frac{M}{\kappa T_{\mathrm{r}}}\right)^{\frac{2}{3}},
$$

with $T_{\mathrm{H}}$ being the cosmic temperature at time $t_{\mathrm{H}}$.

After the end of the second inflationary stage, the system settles in the SUSY vacuum and a linear combination of the $U(1)_{B-L}$ gauge symmetry and the unbroken $U(1)$ subgroup of $S U(2)_{\mathrm{R}}$ breaks spontaneously leading to the production of local cosmic strings. These strings, if they survived after recombination, could have a small contribution to the CMBR power spectrum which is parametrized [21] by the dimensionless string tension $G \mu_{\mathrm{s}}$, where $G$ is Newton's constant and $\mu_{\mathrm{s}}$ is the string tension, i.e. the energy per unit length of the string. For local strings in the Abelian Higgs model in the Bogomol'nyi limit, the string tension is [21]

$$
\mu_{\mathrm{s}}=4 \pi|\langle H\rangle|^{2}
$$

where $\langle H\rangle$ is the VEV of $H$. Although the strings in our model are more complicated, we think that the above estimate is good enough for our purposes here.

It is important that the strings, in our case, do not survive after recombination, but decay well before it. So they do not affect the CMBR. The reason is that they are open strings connecting monopoles to antimonopoles. This can be understood by simply realizing that the breaking of $S U(2)_{\mathrm{R}} \times U(1)_{B-L}$ to $U(1)_{Y}$ by $\langle H\rangle$ and $\langle\bar{H}\rangle$ is similar to the breaking of the electroweak gauge group and, thus, cannot lead to any topologically stable monopoles or strings - for a more detailed explanation of this fact, see Ref. [22]. This breaking can only lead to the formation of topologically unstable dumbbell configurations [23] consisting of an open string connecting a monopole to an antimonopole.

At any time after their formation, the strings look like random walks with a step of the order of the particle horizon connecting monopoles to antimonopoles [24] - to describe the evolution of this string network, we will follow closely this reference. As argued in Ref. [24], at all times before the entrance of monopoles into the horizon, there is of the order of one string segment per horizon and, thus, the ratio of the energy density $\rho_{\mathrm{s}}(t)$ of the string network to the total energy density $\rho_{\text {tot }}(t)$ of the universe remains practically constant. At $t_{\mathrm{H}}$, there is approximately one monopole-antimonopole pair per horizon connected by an almost straight string segment of the size of the horizon. The energy density $\rho_{\mathrm{s}}\left(t_{\mathrm{H}}\right)$ of the strings at $t_{\mathrm{H}}$ is then $\sim 3 G \mu_{\mathrm{s}} / 2 t_{\mathrm{H}}^{2}$. After this time, more and more string segments enter the horizon, but the length of each segment remains 
constant. So the system of string segments behaves like pressureless matter. As a consequence, $\rho_{\mathrm{s}}(t) \sim 3 G \mu_{\mathrm{s}} / 2\left(t_{\mathrm{H}} t^{3}\right)^{1 / 2}$ and the 'relative string energy density'

$$
\frac{\rho_{\mathrm{s}}(t)}{\rho_{\gamma}(t)} \sim 2 G \mu_{\mathrm{s}}\left(\frac{t}{t_{\mathrm{H}}}\right)^{\frac{1}{2}}
$$

$\left(\rho_{\gamma}(t)\right.$ is the 'photon' energy density) increases with time - note that in a radiation dominated universe $\rho_{\gamma}(t)=\rho_{\text {tot }}(t)$. The strings decay at cosmic time [25]

$$
t_{\mathrm{d}} \sim\left(\Gamma G \mu_{\mathrm{s}}\right)^{-1} 2 t_{\mathrm{H}}
$$

with $\Gamma \sim 50$ by emitting gravity waves with energy density $\rho_{\mathrm{gw}}\left(t_{\mathrm{d}}\right)$ at production given by

$$
\frac{\rho_{\mathrm{gw}}\left(t_{\mathrm{d}}\right)}{\rho_{\gamma}\left(t_{\mathrm{d}}\right)} \sim 2\left(\frac{2}{\Gamma}\right)^{\frac{1}{2}}\left(G \mu_{\mathrm{s}}\right)^{\frac{1}{2}} .
$$

Note that this is also the maximal relative string energy density.

Taking the lower value of the number of e-foldings and $p=2$, Eq. (5.2) gives, for the two numerical examples presented, $t_{\mathrm{H}} \sim 4.8 \times 10^{-7} \mathrm{sec}$ and $1.04 \times 10^{-4} \mathrm{sec}$, respectively. Consequently, the strings enter the horizon well before big bang nucleosynthesis. Their decay time is $t_{\mathrm{d}} \sim 6 \times 10^{-2} \mathrm{sec}$ and $5.5 \mathrm{sec}$, in the two cases, as one can infer from the corresponding dimensionless string tensions

$$
G \mu_{\mathrm{s}}=\frac{|\langle H\rangle|^{2}}{2} \simeq \frac{m M}{2 \lambda} \simeq 3.19 \times 10^{-7} \text { and } 7.57 \times 10^{-7}
$$

So the strings decay well before recombination and, thus, do not affect the CMBR. Their maximal relative energy density is $\sim 2.3 \times 10^{-4}$ and $3.5 \times 10^{-4}$ for our two examples and, thus, the strings remain always subdominant. In particular, they do not disturb nucleosynthesis.

Had the strings survived until now, an upper bound would have to be imposed on $G \mu_{\text {s }}$ to keep their contribution to the CMBR power spectrum at an acceptable level. For the Abelian-Higgs field theory model, this bound is [26]

$$
G \mu_{\mathrm{s}} \lesssim 3.2 \times 10^{-7}
$$

In our first numerical example, $G \mu_{\mathrm{s}}$ almost saturates this bound, but violates the recent bound [27]

$$
G \mu_{\mathrm{s}} \lesssim 3.3 \times 10^{-8}
$$

from pulsar timing arrays, which also holds for strings surviving until the present time. Our second numerical example violates both the bounds in Eqs. (5.8) and (5.9). Thus, both our examples are only possible because the strings decay sufficiently early to gravity waves.

The ratio of the energy density of the gravity waves produced by the strings to that of the photons at the present time $t_{0}$ can be found from Eq. (5.6) to be - cf. Ref. [28] -

$$
\frac{\rho_{\mathrm{gw}}\left(t_{0}\right)}{\rho_{\gamma}\left(t_{0}\right)} \sim 2\left(\frac{2}{\Gamma}\right)^{\frac{1}{2}}\left(G \mu_{\mathrm{s}}\right)^{\frac{1}{2}}\left(\frac{3.9}{10.75}\right)^{\frac{4}{3}}
$$


and their present abundance is given by

$$
\Omega_{\mathrm{gw}} h^{2}\left(t_{0}\right) \sim\left(\frac{\rho_{\mathrm{gw}}\left(t_{0}\right)}{\rho_{\gamma}\left(t_{0}\right)}\right)\left(\frac{\rho_{\gamma}\left(t_{0}\right)}{\rho_{\mathrm{c}}\left(t_{0}\right)}\right) h_{0}^{2},
$$

where $\rho_{\mathrm{c}}\left(t_{0}\right)$ is the present critical energy density of the universe and $h_{0} \simeq 0.7$ is the present value of the Hubble parameter in units of $\mathrm{km} \mathrm{sec}^{-1} \mathrm{Mpc}^{-1}$. As it turns out $\Omega_{\mathrm{gw}} h^{2}\left(t_{0}\right) \sim 2.2 \times 10^{-9}$ and $3.4 \times 10^{-9}$ for our two numerical examples, respectively. The frequency $f\left(t_{\mathrm{d}}\right)$ of these gravitational waves at production must be $\sim t_{\mathrm{H}}^{-1}$ since the length of the decaying strings is $\sim 2 t_{\mathrm{H}}$ [25]. The present value of this frequency is then

$$
f\left(t_{0}\right) \sim t_{\mathrm{H}}^{-1}\left(\frac{t_{d}}{t_{\mathrm{eq}}}\right)^{\frac{1}{2}}\left(\frac{t_{\mathrm{eq}}}{t_{0}}\right)^{\frac{2}{3}},
$$

where $t_{\mathrm{eq}}$ is the equidensity time at which matter starts dominating the universe. For the two numerical examples, this frequency turns out to be $\sim 1.1 \times 10^{-4} \mathrm{~Hz}$ and $4.7 \times 10^{-6} \mathrm{~Hz}$, respectively. We see that these frequencies are too high to yield any restriction from CMBR considerations [28]. Also, they are well above the range probed by the pulsar timing array observations [29] and, thus, the recent bound [27] from pulsars does not apply to our case. However, the frequency of the gravity waves in our first numerical example lies marginally within the range to be probed by the future space-based laser interferometer gravitational-wave observatories such as eLISA/NGO [30]. We conclude that the monopole-string system decays early enough without causing any trouble, but the gravity waves that it generates may be probed by future space-based laser interferometer observations.

\section{Conclusions}

We considered a reduced version of the extended SUSY PS model of Ref. [12], which was initially constructed for solving the $b$-quark-mass problem of the simplest SUSY PS model with universal boundary conditions. We find that this model can yield a two stage hybrid inflationary scenario predicting values of the tensor-to-scalar ratio of the order of few $\times 10^{-2}$. For the values of the parameters chosen, the model in global SUSY possesses practically two classically flat directions: the trivial and the semi-shifted one. We have shown that the SUGRA corrections stabilize the trivial path, which can then support a first stage of inflation with a limited number of e-foldings. The obtained value of tensor-to-scalar ratio can be appreciable as a result of mild SUGRA corrections combined with strong radiative corrections to the inflationary potential, while the scalar spectral index remains acceptable.

The extra e-foldings required for solving the horizon and flatness problems of the standard hot big bang cosmological model are generated by a second stage of inflation along the semi-shifted path, where the gauge group $U(1)_{B-L}$ is unbroken. This is possible since the SURGA corrections to the potential on the semi-shifted path remain mild and this path is almost orthogonal to the trivial one and, thus, is not affected by the strong radiative corrections to the potential on the trivial path.

After the termination of the first inflationary stage, magnetic monopoles are formed. Subsequently, at the end of the second stage of inflation, cosmic strings are produced connecting 
these monopoles to antimonopoles. At later times, the monopoles enter the horizon and the stringmonopole system decays into gravity waves well before recombination without leaving any trace in the CMBR. The resulting gravity waves, however, may be measurable in the future.

The baryon asymmetry of the universe can, in principle, be generated by non-thermal leptogenesis [31]. At reheating, the inflaton system decays into right-handed neutrino superfields, which subsequently decay out of equilibrium into light matter generating a primordial lepton asymmetry. This asymmetry is then partly turned into the observed baryon asymmetry of the universe by electroweak sphaleron effects. A detailed discussion of this mechanism within a particle physics model based on the same left-right symmetric gauge group as our present model, but with some differences, can be found in Ref. [32].

\section{References}

[1] P.A.R. Ade et al. [BICEP2 Collaboration], Detection of B-Mode polarization at degree angular scales by BICEP2, Phys. Rev. Lett. 112 (2014) 241101 [arXiv: 1403.3985 ].

[2] R. Flauger, J.C. Hill, and D.N. Spergel, Toward an understanding of foreground emission in the BICEP2 region, J. Cosmol. Astropart. Phys. 08 (2014) 039 [arXiv: 1405.7351 ]; M. Cortês, A.R. Liddle, and D. Parkinson, Tensors, BICEP2, prior dependence, and dust, Phys. Rev. D 92 (2015) 063511 [arXiv:1409.6530].

[3] M.J. Mortonson and U. Seljak, A joint analysis of Planck and BICEP2 B modes including dust polarization uncertainty, J. Cosmol. Astropart. Phys. 10 (2014) 035 [arXiv: 1405.5857 ]; C. Cheng, Q.G. Huang, and S. Wang, Constraint on the primordial gravitational waves from the joint analysis of BICEP2 and Planck HFI $353 \mathrm{GHz}$ dust polarization data, ibid. 12 (2014) 044 [arXiv:1409.7025]; L. Xu, Detecting primordial gravitational waves signal from BICEP2 and Planck HFI 353GHz dust polarization, arXiv: 1409.7870.

[4] P.A.R. Ade et al. [BICEP2/Keck and Planck Collaborations], Joint analysis of BICEP2/Keck Array and Planck data, Phys. Rev. Lett. 114 (2015) 101301 [arXiv: 1502 . 00612].

[5] E.J. Copeland, A.R. Liddle, D.H. Lyth, E.D. Stewart, and D. Wands, False vacuum inflation with Einstein gravity, Phys. Rev. D 49 (1994) 6410 [astro-ph/ 9401011 ]; G.R. Dvali, Q. Shafi, and R.K. Schaefer, Large scale structure and supersymmetric inflation without fine tuning, Phys. Rev. Lett. 73 (1994) 1886 [hep-ph / 9406319 ]; G. Lazarides, R.K. Schaefer, and Q. Shafi, Supersymmetric inflation with constraints on superheavy neutrino masses, Phys. Rev. D 56 (1997) 1324 [hep-ph/9608256]; G. Lazarides, Q. Shafi, and N.D. Vlachos, Supersymmetric inflation, baryogenesis and $v_{\mu}-v_{\tau}$ oscillations Phys. Lett. B 427 (1998) 53 [hep-ph/9706385].

[6] G. Lazarides, Supersymmetric hybrid inflation, in proceedings of NATO ASI 2000: Recent Developments in Particle Physics and Cosmology, NATO Sci. Ser. II 34 (2001) 399 [hep-ph/0011130].

[7] G. Lazarides and C. Panagiotakopoulos, Smooth hybrid inflation, Phys. Rev. D 52 (1995) R559 [hep-ph/9506325].

[8] P.A.R. Ade et al. [Planck Collaboration], Planck 2015 results. XIII. Cosmological parameters, arXiv:1502.01589.

[9] C. Panagiotakopoulos, Blue perturbation spectra from hybrid inflation with canonical supergravity, Phys. Rev. D 55 (1997) R7335 [hep-ph / 9702433 ]; A.D. Linde and A. Riotto, Hybrid inflation in supergravity, ibid. 56 (1997) R1841 [hep-ph/ 970320 9]; V.N. Şenoğuz and Q. Shafi, Testing 
supersymmetric grand unified models of inflation, Phys. Lett. B 567 (2003) 79 [hep-ph/ 030508 9]; GUT scale inflation, nonthermal leptogenesis, and atmospheric neutrino oscillations, ibid. 582 (2004) 6 [hep-ph/ 0309134$].$

[10] G. Lazarides and A. Vamvasakis, Standard-smooth hybrid inflation, Phys. Rev. D 76 (2007) 123514 [arXiv:0709.3362].

[11] C. Panagiotakopoulos and N. Tetradis, Two stage inflation as a solution to the initial condition problem of hybrid inflation, Phys. Rev. D 59 (1999) 083502 [hep-ph/9710526]; G. Lazarides and N. Tetradis, Two stage inflation in supergravity, ibid. 58 (1998) 123502 [hep-ph/9802242].

[12] M.E. Gomez, G. Lazarides, and C. Pallis, Yukawa quasi-unification, Nucl. Phys. B 638 (2002) 165 [hep-ph/0203131].

[13] G. Lazarides and C. Panagiotakopoulos, Large $\tan \beta$ from $S U(2)_{R}$ gauge symmetry, Phys. Lett. $B$ 337 (1994) 90 [hep-ph/9403316]; S. Khalil, G. Lazarides, and C. Pallis, Cold dark matter and $b \rightarrow s \gamma$ in the Hořava-Witten theory, ibid. $\mathbf{5 0 8}$ (2001) 327 [hep-ph/ 0005021$].$

[14] B. Ananthanarayan, G. Lazarides, and Q. Shafi, Top-quark-mass prediction from supersymmetric grand unified theories, Phys. Rev. D 44 (1991) 1613; Radiative electroweak breaking and sparticle spectroscopy with $\tan \beta \simeq m_{t} / m_{b}$, Phys. Lett. B 300 (1993) 245; M.E. Gomez, G. Lazarides, and C. Pallis, Supersymmetric cold dark matter with Yukawa unification, Phys. Rev. D 61 (2000) 123512 [hep-ph/9907261].

[15] G. Lazarides and C. Panagiotakopoulos, Gravitational waves from double hybrid inflation, arXiv: 1505.04926 .

[16] G. Lazarides, I.N.R. Peddie, and A. Vamvasakis, Semi-shifted hybrid inflation with B-L cosmic strings, Phys. Rev. D 78 (2008) 043518 [arXiv: 0804 . 3661].

[17] R. Jeannerot, S. Khalil, and G. Lazarides, New shifted hybrid inflation, J. High Energy Phys. 07 (2002) 069 [hep-ph/ 020724 4].

[18] C. Panagiotakopoulos, Hybrid inflation in supergravity with $(S U(1,1) / U(1))^{m}$ Kähler manifolds, Phys. Lett. B 459 (1999) 473 [hep-ph/9904284]; Realizations of hybrid inflation in supergravity with natural initial conditions, Phys. Rev. D 71 (2005) 063516 [hep-ph/ 0411143 ].

[19] G. Lazarides and N.D. Vlachos, Initial conditions for supersymmetric inflation, Phys. Rev. D 56 (1997) 4562 [hep-ph/9707296].

[20] G. Lazarides and Q. Shafi, Extended structures at intermediate scales in an inflationary cosmology, Phys. Lett. B 148 (1984) 35.

[21] N. Bevis, M. Hindmarsh, M. Kunz, and J. Urrestilla, CMB power spectrum contribution from cosmic strings using field-evolution simulations of the Abelian Higgs model, Phys. Rev. D 75 (2007) 065015 [astro-ph/ 0605018 ]; CMB polarization power spectra contributions from a network of cosmic strings, ibid. 76 (2007) 043005 [arXiv : 0704 . 3800]; Fitting CMB data with cosmic strings and inflation, Phys. Rev. Lett. 100 (2008) 021301 [astro-ph/ 0702223].

[22] G. Lazarides, R. Ruiz de Austri, and R. Trotta, Constraints on a mixed inflaton and curvaton scenario for the generation of the curvature perturbation, Phys. Rev. D 70 (2004) 123527

[hep-ph/0409335].

[23] Y. Nambu, Strings, monopoles and gauge fields, Phys. Rev. D 10 (1974) 4262; String-like configurations in the Weinberg-Salam theory, Nucl. Phys. B 130 (1977) 505. 
[24] G. Lazarides, Q. Shafi, and T. Walsh, Cosmic strings and domains in unified theories, Nucl. Phys. B 195 (1982) 157.

[25] A.Vilenkin and E.P.S. Shellard, Cosmic strings and other topological defects, Cambridge University Press, Cambridge 2000.

[26] P.A.R. Ade et al. [Planck Collaboration], Planck 2013 results. XXV. Searches for cosmic strings and other topological defects, Astron. Astrophys. 571 (2014) A25 [arXiv:1303. 5085].

[27] Z. Arzoumanian et al. [NANOGrav Collaboration], The NANOGrav nine-year data set: limits on the isotropic stochastic gravitational wave background, arXiv: 1508.03024.

[28] M. Maggiore, Gravitational wave experiments and early universe cosmology, Phys. Rep. 331 (2000) 283 [gr-qc/9909001].

[29] X.-J. Liu, W. Zhao, Y. Zhang, and Z.-H. Zhu, Detecting relic gravitational waves by pulsar timing arrays: effects of cosmic phase transitions and relativistic free-streaming gases, arXiv: 1509.03524.

[30] P. Amaro-Seoane et al., eLISA/NGO: astrophysics and cosmology in the gravitational-wave millihertz regime, GW Notes 6 (2013) 4 [arXiv: 1201 . 3621].

[31] G. Lazarides and Q. Shafi, Origin of matter in the inflationary cosmology, Phys. Lett. B 258 (1991) 305.

[32] R. Armillis, G. Lazarides, and C. Pallis, Inflation, leptogenesis, and Yukawa quasiunification within a supersymmetric left-right model, Phys. Rev. D 89 (2014) 065032 [arXiv: 1309 . 6986]. 